\title{
Adapted Wavelet Transform for Twisted Blade Diagnosis in Multi Stage Rotor
}

\author{
Ahmed M. Abdelrhman ${ }^{1,}$, M. Salman Leong ${ }^{2}$, Y.H.Ali ${ }^{3}$, Iftikhar Ahmad ${ }^{1}$, Christina G. Georgantopoulou ${ }^{1}$ and Salah M. Ali ${ }^{2}$ \\ ${ }^{1}$ Department of Mechanical Engineering, School of Engineering, Bahrain Polytechnic, 33349 Isa Town, Kingdom of Bahrain. \\ ${ }^{2}$ Institute of Noise and Vibration, Universiti Teknologi Malaysia, Jalan Sultan Yahya Petra, 54100 Kuala Lumpur, Malaysia. \\ ${ }^{3}$ Department of Refrigeration and Air-conditioning, Technical College of Mosul, Northern Technical University, Mosul, Iraq.
}

\begin{abstract}
This paper studies the diagnosis of twisted blade in a multi stages rotor system using adapted wavelet transform and casing vibration. The common detection method (FFT) is effective only if sever blade faults occurred while the minor faults usually remain undetected. Wavelet analysis as alternative technique is still unable to fulfill the fault detection and diagnosis accurately due to its inadequate time-frequency resolution. In this paper, wavelet is adapted and its time-frequency is improved. Experimental study was undertaken to simulate multi stages rotor system. Results showed that the adapted wavelet analysis is effective in twisted blade diagnosis compared to the conventional one.
\end{abstract}

\section{Introduction}

Most of the failure cases in turbomachinery is reported to be due blade failure. Therefore, an extensive research has been conducted in the field of blade condition monitoring techniques and signal analysis methods for blade faults detection and diagnosis. The intension was to detect the faults at the early stage before the catastrophic failure take place. An extensive review on this subject can be found in ref [1-3]. Blade deformation such as blade twisting, creep, erosion, and corrosion are common faults that occurred in turbomachinery. Blade twisting could cause obstruction in the flow of a machine, and therefore could be detected by measuring the pressure field around the blades. This method has been proposed by Mathioudakis et al. [4], and Dedoussis et al. [5]. As showed by Aretakis [6], the distortion of pressure signals around the blades could indicate the occurrence of twisted blade. Although most of these methods were shown to be effective in varying degree for blade faults diagnosis, a major difficulty relates to its use under practical field conditions and the huge number of sensors required to be installed inside the machine. For application under practical field condition, vibration based methods inevitably represents the most

\footnotetext{
Corresponding author: ahmedrabak@gmail.com
}

readily and widely used methods for blade fault diagnosis. This is due to the fact that vibration signals are directly related to the response of the machine structural dynamics due to changes in working conditions within the machine. Vibration signals are conventionally analysed with Fourier analysis to obtain the vibration spectrum, with blade passing frequency (BPF) being the most commonly used primary parameter for detection of blade faults. Relative changes in blade passing frequency and its harmonic amplitude could provide useful information upon which blade faults could be detected [7-10]. Blade faults are however difficult to be detected during normal machine operations, and vibration analysis can only detect blade faults if severe damage occurs at the blade $[11,12]$, where minor blade faults not so readily detected. Common vibration diagnosis techniques are based on frequency domain analysis with changes to be detected in the vibration spectra (i.e. escalating BPF amplitude). The vibration amplitude is deemed an indicator of severe blade damage occurrence and used for assessment of any imminent failure. Field experiences however had been shown that blade faults do not result in significant increase in the overall vibration amplitude as the overall vibration amplitude are dictated by the synchronous unbalance response of the machine. 
For blade faults diagnosis, wavelet analysis it's been used recently [13] to provide better visualization of the blade conditions in comparison to vibration spectra. However, wavelet itself have its own limitation. In this context the current author investigated the vibration characteristics of the multi-stages rotor system by examining the limitations and difficulties in conventional wavelets in analysing the multi-stage rotor signal [14]. Conventional wavelet transform and other time-frequency analysis method such as Short Time Fourier Transform (STFT), Wigner-Ville distribution (WVD) and Choi-Williams distribution (CWD) has shown to be have some limitations and lack in its resolution hence it's incapable to accurately display the faults characteristics and location [15]. Therefore, the currents author tried to adapt the wavelet transform using reassignment technique for better blade twisting fault diagnosis. More details about the wavelet reassignment and adaptation can be found in ref [16], this includes highlight of the function of the newly proposed wavelet and how the wavelet resolution improved for blade fault diagnosis in multi-stage rotor system.

Current research in the open literature on the diagnosis of the twisted blade revealed that it is still not possible to identify the exact location of the twisted blade in raw using vibration spectral analysis [17]. Therefore, the objective of this study was to diagnose the blade twist fault in multi stage rotor system and identify the exact position of twisted blade in the row using adapted wavelet technique.

\section{Experimental Study}

A test rig was constructed to conduct the experimental study with the aim of both replicating realistic blade fault under controlled conditions and providing detailed measurements on blade twist fault conditions. The designed experimental test rig consisted of a single shaft connected to an electric motor with a coupling device and three rows of rotor blades, with each row having 8,11 and 13 number of blades arranged into the first row to the third respectively. The blade twist fault was induced into the test rig to provide experimental vibration data for the blade twist faults. Faults were induced at stage 1 blade number 4 , stage 2 blade number 5 and stage 3 blade number 8 . Then, accelerometers were mounted on the rotor casing to pick up the vibration signals. Once per revolution trigger (tachometer) was also installed to track the operating speed and to provide speed control. Fig. 1 is showing a photograph of the rotor system.

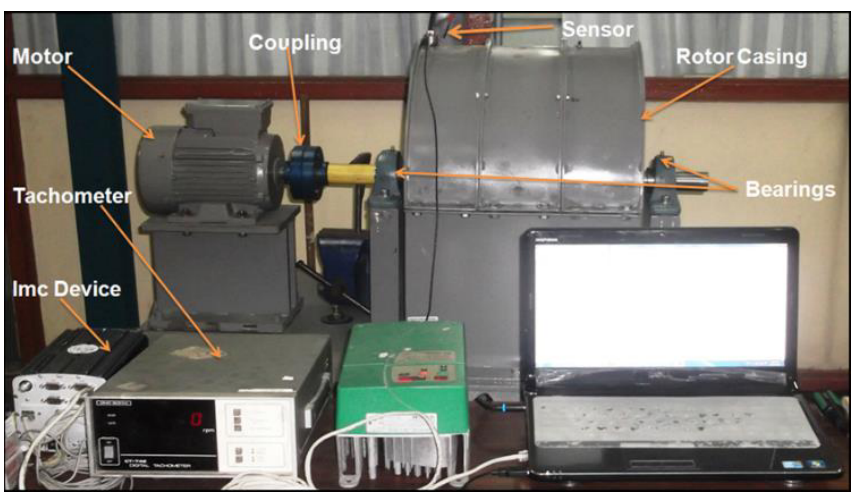

Fig. 1 .Experimental Test Rig Assembly

The below flow chart in Fig. 2. shows the schematic diagram of the proposed method and the steps required to apply it along with the data reduction procedures.

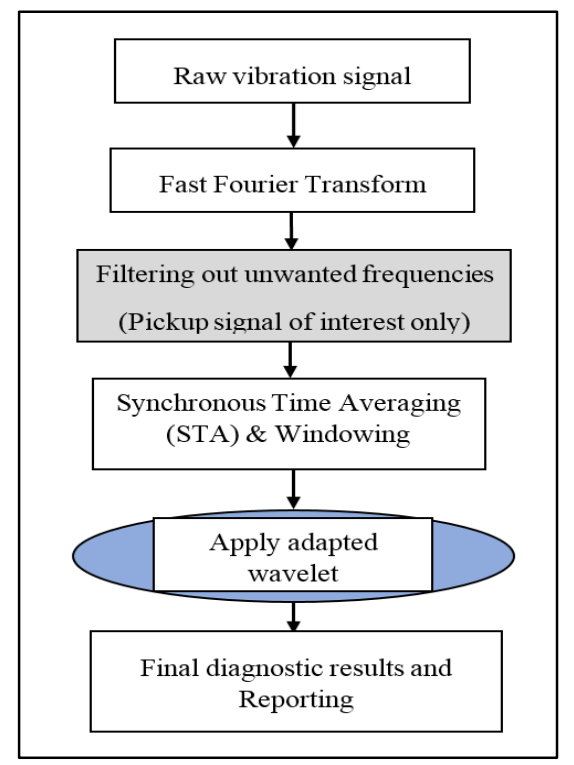

Fig. 2. Schematic diagram of the proposed method

\section{Experimental Results and Discussion}

\subsection{Vibration Spectra}

Fig. 3. presents the vibration spectra of blade twisting fault conditions. It could be observed that there were no obvious changes in the vibration spectra as compared to the baseline data. The only changes noted were sidebands and other multiple harmonics across the vibration spectra. A blade twisting fault would be difficult to be detected based on vibration spectra. 


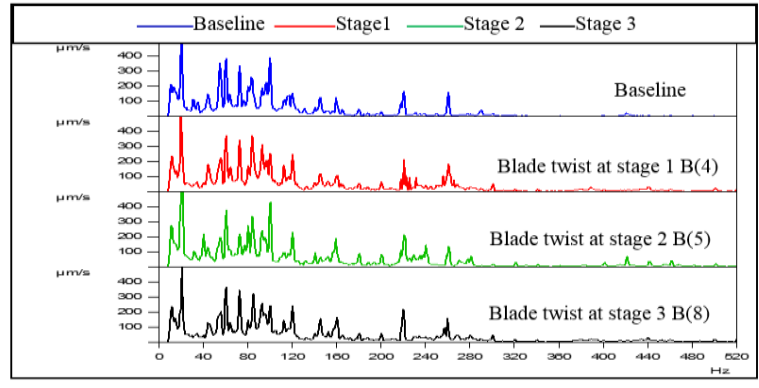

Fig. 3. Vibration Spectra Analysis

\subsection{Rotor Wavelet Maps}

Fig. (4, 5(a). 5(b) and 5(c) shows the wavelet maps of the induced blade twisting faults at the rotor different stages. Rotor dynamic wavelet maps of the conventional wavelet Fig. 4, did not demonstrate any evidences for blade twisting faults when compared to the baseline data, where both conditions had similar patterns. This was indeed due to the lack of resolution in both time and frequency domains. Changes in the rotor wavelet maps due to the blade twisting were evident when ahmedrabak_time wavelet [16] was derived for the blades faulty signals. The presence and the locations of twisted blades were clearly evident. Presence of blade twisting fault in the rotor wavelet maps was demonstrated as amplitude decrement and distortion in the position of twisted blades and the surrounded blades (refer to Figs (5(a), 5(b) and 5(c)). The advantages of using ahmedrabak time wavelet over the conventional wavelet analysis and vibration spectra were clearly demonstrated and hence, location of twisted blades could be identified and number of twisted blades could be estimated.

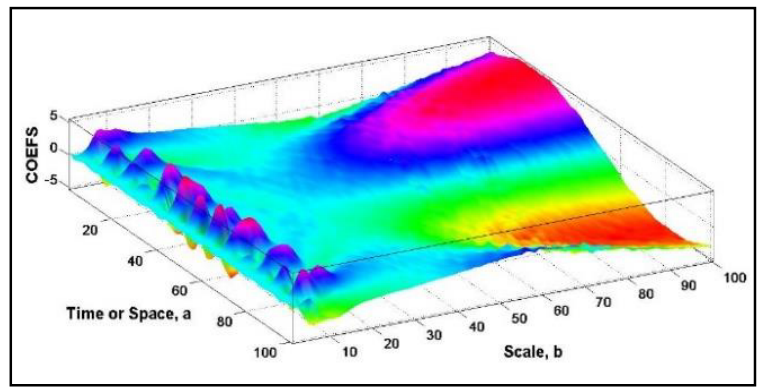

Fig. 4. Conventional wavelet rotor map

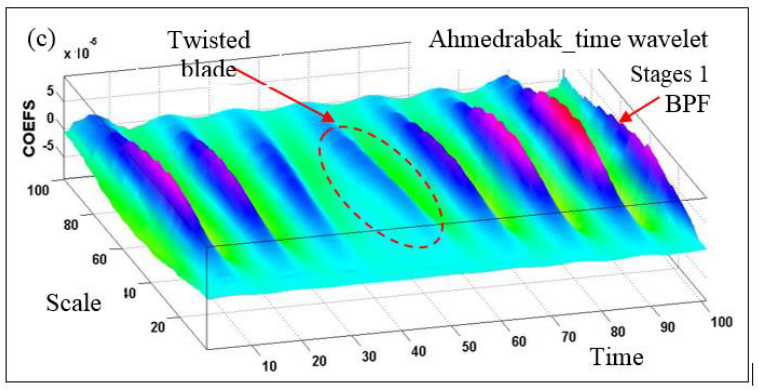

Fig. 2(a). Wavelet map for blade 4 twist fault

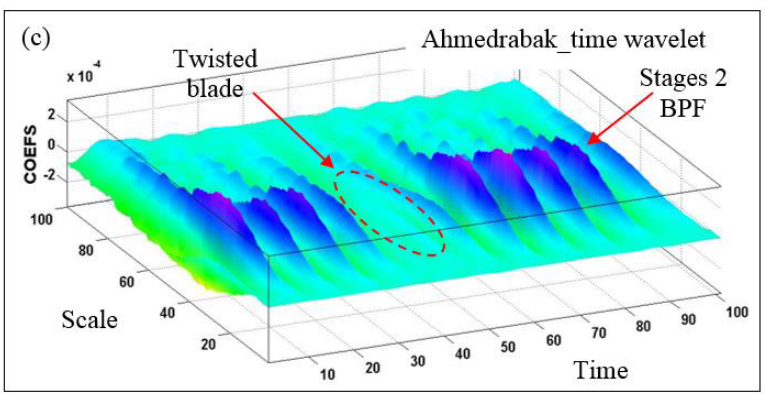

Fig. 5(b). Wavelet map for blade 5 twist fault

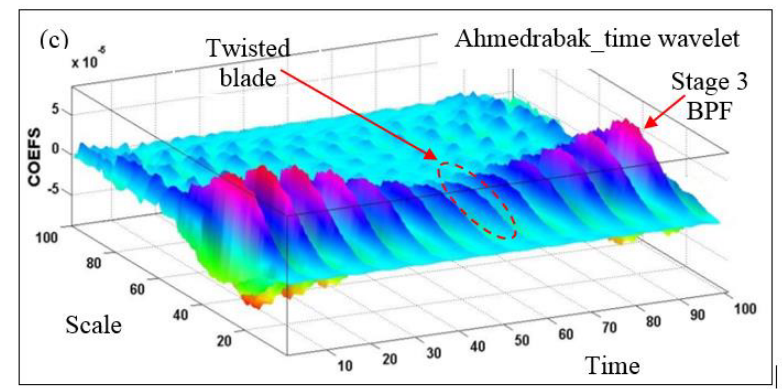

Fig. 5(c). Wavelet map for blade 8 twist fault

\section{Conclusion}

In this paper, a new method for blade twisting diagnosis is examined. Application of the proposed method in the blade twisting diagnosis in multi-stage rotor system showed that the newly formulated technique provided greater diagnostic information than the existing vibration analysis techniques in particular FFT and conventional wavelet analysis transform. The proposed technique has higher resolution in time than conventional wavelet analysis method, allowing small-short frequency's amplitude modulation and small time signal alteration in the blade passing frequency (BPF) signals with faults to be obvious and clearly seen. This was demonstrated for the identification of the exact location of the twisted blade in raw and the number of faulty blades. 


\section{References}

[1] A. M. Abdelrhman, M. S. Leong, S. A. M. Saeed, and S. M. Al Obiadi, "A Review of Vibration Monitoring as a Diagnostic Tool for Turbine Blade Faults," Appl. Matt. Res, vol. 229, pp. 1459-1463, 2012.

[2] A. M. Abdelrhman, L. M. Hee, M. S. Leong, and S. Al-Obaidi, "Condition Monitoring of Blade in Turbomachinery: A Review," Adv. Mech. Eng, vol. 2014, p. 10, 2014.

[3] K. H. Hui, L. M. Hee, M. S. Leong, and A. M. Abdelrhman, "Time-Frequency Signal Analysis in Machinery Fault Diagnosis," in Adv Mat Res, 2014, pp. 41-45.

[4] K. Mathioudakis, A. Papathanasiou, E. Loukis, and K. Papailiou, "Fast response wall pressure measurement as a means of gas turbine blade fault identification," JPGC - Pwr, vol. 113, pp. 269-275, 1991.

[5] V. Dedoussis, K. Mathioudakis, and K. Papailiou, "Numerical simulation of blade fault signatures from unsteady wall pressure signals," $J P G C$ - Pwr, vol. 119, pp. 362-369, 1997.

[6] N. Aretakis and K. Mathioudakis, "Wavelet analysis for gas turbine fault diagnostics," JPGC - Pwr, vol. 119, 1997.

[7] H. Simmons, "A non-intrusive method for detecting HP blade resonance," ASME Paper No, 1986.

[8] H. Simmons, "A Non-Intrusive Method for Detecting HP Turbine Blade Resonance," ASME Paper No, vol. 86, 1986.

[9] P. Parge, Trevillion, B., Carle, P, "Machinery Interactive Display and Analysis System Description and Applications," in Proceedings of the First International Machinery Monitoring and Diagnostic Conference, Las Vegas, Nevada Sept 11-14, 1989, pp. 176-182.

[10] P. Parge, Trevillion, B., Carle, P, "Non-Intrusive Vibration Monitoring for Turbine Blade Reliability," in Proceedings of Second International Machinery Monitoring and Diagnostic Conference, Los Angeles, California, Oct 22-25, 1990, pp. Pp. 435- 446.

[11] M. H. Lim and M. Salman Leong, "Diagnosis for loose blades in gas turbines using wavelet analysis," JPGC - Pwr, vol. 127, pp. 314-322, 2005.

[12] K. Mathioudakis, E. Loukis, and K. D. Papailiou, "Casing vibration and gas turbine operating conditions," JPGC - Pwr, vol. 112, pp. 478-485, 1990.

[13] A. M. Abdelrhman, M. S. Leong, L. M. Hee, and K. H. Hui, "Vibration Analysis of Multi Stages Rotor for Blade Faults Diagnosis," Adv Mat Res, vol. 845, pp. 133-137, 2014.
[14] Ahmed.M.Abdelrhman, M. S. Leong, L. M. Hee, and W. K. Ngui, "Application of Wavelet Analysis in Blade Faults Diagnosis for MultiStages Rotor System," Appl. Matt. Res, 2013.

[15] A. M. Abdelrhman, M. S. Leong, Y. M. Hamdan, and K. H. Hui, "Time Frequency Analysis for Blade Rub Detection in Multi Stage Rotor System," in Appl. Matt. Res, 2015, pp. 95-99.

[16] A. M. Abdelrhman, M. S. Leong, L. M. Hee, and W. K. Ngui, "A Comparative Study of Reassigned Conventional Wavelet Transform for Machinery Faults Detection," in Appl. Matt. Res, 2015, pp. 90-94.

[17] W. K. Ngui, M. Salman Leong, L. Hee, and A. M. Abdelrhman, "Detection of Twisted Blade in Multi Stage Rotor System," in Appl. Matt. Res, 2015, pp. 144-148. 